\title{
Programmable Diffractive Optical Elements with Applicability in Ophthalmic Optics
}

\section{Elementos ópticos difractivos programables con aplicabilidad en óptica oftálmica}

\author{
Lenny A. Romero ${ }^{1 \mathrm{P}^{*}}$, María S. Millán ${ }^{2 \mathrm{~S}}$. \\ 1. Facultad de Ciencias Basicas, Universidad Tecnológica de Bolivar, Km 1 vía Turbaco, \\ Cartagena, Colombia \\ 2. Dep. Optics and Optometry, Universitat Politècnica de Catalunya BARCELONATECH, C/ Violinista Vellsolà, \\ 37, 08222 Terrassa (Barcelona), Spain \\ (*) E-mail: lromero@unitecnologica.edu.co \\ S: SEDOPTICA member / P: SRCO member
}

DOI: 10.7149/OPA.50.1.49509

\begin{abstract}
:
This paper presents results of the research related with the development of the doctoral thesis of Lenny Alexandra Romero Pérez. This work covers the design, the characterization and the analysis of several programmable diffractive optical elements (PDOEs) such as: Fresnel lenses, multifocal lenses, integrated combinations of phase-masks with lenses, and DOEs with extended depth of focus (EDOF) like the Light Sword optical element and the Peacock Eye. This has been pursued to address several problems of human vision, like myopia, hyperopia, astigmatism, and presbyopia, by means of the implementation of such DOEs on a Holoeye Liquid Crystal on Silicon HEO 1080P spatial light modulator. We have developed and implemented several algorithms for generating the necessary optical elements to compensate the different ametropies. Simulation and experimental results demonstrate that several of the considered DOEs have the sufficient imaging performance and thus the potential for compensating ametropies and presbyopia.
\end{abstract}

Key words: extended depth of focus, diffractive optical elements; ophthalmic optics, visual optics, spatial light modulator.

\section{RESUMEN:}

Este trabajo presenta resultados de la investigación enmarcada en la tesis doctoral realizada por la doctora Lenny Alexandra Romero Pérez, la cual fue enfocada en el diseño, caracterización y análisis de diversos elementos ópticos difractivos programables (PDOEs por sus siglas en inglés) programables tales como: lentes de Fresnel, lentes multifocales, combinaciones integradas de máscaras de fase con lentes y elementos con capacidad de extender la profundidad de foco (EDOF del inglés, extended depth of focus), como los elementos difractivos llamados espada de luz y ojo de pavo. Esta tesis busca solventar por medio del uso de DOEs en un modulador espacial de luz diversos problemas de la visión humana, como la miopía, hipermetropía, astigmatismo y la presbicia. La implementación se llevó a cabo en un modulador Holoeye LCoS (del inglés Liquid Crystal on Silicon) de 1080P. Se han desarrollado e implementado distintos algoritmos para la generación de los elementos ópticos necesarios para la compensación de las ametropías. Los resultados obtenidos mediante pruebas de simulación y experimentación permiten contrastar las propiedades de los DOEs analizados y destacar aquellos que por su funcionalidad en la compensación de ametropías pueden alcanzar una mayor aplicabilidad. En este artículo presentamos los resultados más relevantes obtenidos en esta investigación.

Palabras clave: elementos ópticos difractivos programables, profundidad de foco extendido, óptica oftálmica, óptica visual, modulador espacial de luz. 


\section{REFERENCES AND LINKS / REFERENCIAS Y ENLACES}

[1] V. Laude, "Twisted-nematic liquid-crystal pixelated active lens," Opt. Commun. 153, 134-152, (1998). https://doi.org/10.1016/S0030-4018(98)00143-6

[2] M. S. Millan, J. Otón, and E. Perez-Cabre, "Dynamic compensation of chromatic aberration in a programmable diffractive lens," Opt Express, 14, 9103-9112, (2006). https://doi.org/10.1364/OE.14.009103

[3] L. A. Romero, M. S. Millan, and E. Perez-Cabre, "Lente programable multifocal: Combinación coaxial y multieje," Opt. Pura Apl., 43, 101-112, (2010).

[4] L. A. Romero, M. S. Millan, Z. Jaroszewicz, and A. Kolodziejczyk, "Double peacock eye optical element for extended focal depth imaging with ophthalmic applications," J Biomed Opt, 17, 046013-046013, (2012).

https://doi.org/10.1117/1.JB0.17.4.046013

[5] L. N. Thibos and A. Bradley, "Use of liquid-crystal adaptive-optics to alter the refractive state of the eye.," Optom Vis Sci, 74, 7, 581-587, (1997). https://doi.org/10.1097/00006324-199707000-00028

[6] G. Li, D. Mathine, P. Valley, P. Äyräs, J. Haddock, M. Giridhar, G. Williby, J. Schwiegerling, G. Meredith, and B. Kippelen, "Switchable electro-optic diffractive lens with high efficiency for ophthalmic applications,” Proc. Natl. Acad. Sci. U.S.A. 103, 6100-6104 (2006). https://doi.org/10.1073/pnas.0600850103

[7] L. A. Romero, M. S. Millan, and E. Perez-Cabre, "Optical implementation of multifocal programmable lens with single and multiple axes,” Journal of Physics: Conference Series, 274, 012050 (2011). https://doi.org/10.1088/1742-6596/274/1/012050

[8] J. Otón, P. Ambs, M. S. Millan, and E. Perez-Cabre, "Multipoint phase calibration for improved compensation of inherent wavefront distortion in parallel aligned liquid crystal on silicon displays," Appl. Opt. 46, 5667-5679, (2007). https://doi.org/10.1364/A0.46.005667

[9] N. Davidson, A. A. Friesem, and E. Hasman, "Holographic axilens: high resolution and long focal depth," Opt. Lett. 16, 523-525, (1991). https://doi.org/10.1364/OL.16.000523

[10] J. Sochacki, S. Bara, Z. Jaroszewicz, and A. Kołodziejczyk, "Phase retardation of the uniform-intensity axilens," Opt. Lett. 17, 7-9, (1992). https://doi.org/10.1364/OL.17.000007

[11] A. Kołodziejczyk, S. Bará, Z. Jaroszewicz, and M. Sypek, "The light sword optical element-a new diffraction structure with extended depth of focus," J. Mod. Opt. 37, 8, 1283-1286, (1990). https://doi.org/10.1080/09500349014551431

[12] Z. Jaroszewicz, A. Kołodziejczyk, D. Mouriz, and J. Sochacki, "Generalized zone plates focusing light into arbitrary line segments,” J. Mod. Opt. 40, 601-612, (1993). https://doi.org/10.1080/09500349314550661

[13] J. Otón, M. S. Millán, and E. Pérez Cabré, "Programmable lens design in a pixelated screen of twistednematic liquid crystal display," Opt. Pura Apl. 38, 47-56, (2005).

[14] J. Otón, M. S. Millan, E. Perez-Cabre, P. García-Martínez, G. Cristóbal, B. Javidi, and S. Vallmitjana, "Imaging characteristics of programmable lenses generated by SLM," AIP Conference Proceedings, 860, 471-480 (2006). https://doi.org/10.1063/1.2361252

[15] R. R. Bennett, "Rabbetts' Clinical Visual Optics,” (1998).

[16] Z. Jaroszewicz, A. Kołodziejczyk, D. Mouriz, and J. Sochacki, "Generalized zone plates focusing light into arbitrary line segments," J. Mod. Opt. 40, 601-612, (1993). https://doi.org/10.1080/09500349314550661

\section{Introduction}

Programmable diffractive lenses, displayed on liquid-crystal pixelated devices are of common use in optical processors and information optics [1]. Very often they are multiplexed to other elements such as filters or holograms so as to implement a complex diffractive optical element [2-4]. Despite their attractive properties of refreshment and flexible design, the application of programmable lenses to ophthalmic optics for ametropia compensation is, however, very limited. Some liquid crystal devices have been 
already proposed for their use as ophthalmic lenses and some prototypes can be found in the literature [5, 6]. They usually have a diffractive design, either displayed on a pixelated device or on a device consisting of other arrays of cells (e.g., with circular symmetry in Ref. [6]). Some practical difficulties arise when considering the ophthalmic application of programmable lenses displayed on liquid-crystal devices: low efficiency, chromatic aberrations (emphasized with diffractive designs), polarized light requirements, etc.

The attractive properties of refreshment and flexible design can be achieved when displaying the programmable diffractive lens (also called Phase Fresnel hologram, kinoform lens, and active lens) on a pixelated liquid crystal screen that acts as a phase-only spatial light modulator. Modern LCoS (liquid crystal on silicon) devices reach $2 \pi$ phase modulation with almost inexistent amplitude coupled modulation, high efficiency, and small pixel pitch (about 8 microns) so that it is possible to generate lenses of power up to 9D (D stands for diopter) for a wavelength of $633 \mathrm{~nm}$. On the other hand, these devices are typically reflective and have a relatively small aperture (e.g. $0.9 \mathrm{~cm} \times 1.5 \mathrm{~cm}$ ), which are clear drawbacks in ophthalmic applications. Other practical limitations are related to the mechanics and electronics requirements of the current state of the art of spatial light modulators (SLMs). But, apart from spectacles, there are other ophthalmic applications that may still be considered, as shown in Ref. [5]. For instance, programmable lenses could be inserted in the eyepieces of optical instruments or in phoropters for optometric assessment of visual acuity. In these cases, the use of diffractive programmable lenses would potentially compensate for the possible refractive error (i.e. ametropia) of the observer (or patient) with similar or even more accuracy than conventional components.

In this work, we explore the potential capability of diffractive lenses and programmable diffractive optics elements (PDOEs) with extended depth of focus (EDOF) displayed on a pixelated LCoS device to quickly determine and compensate for the refractive error of the observer eye. We consider common ametropies, such as myopia, hyperopia, astigmatism and presbyopia. In a first stage, we demonstrate the feasibility of the proposal on an artificial eye in optical bench. The artificial eye, which is initially emmetropic, is converted into ametropic by introducing other lenses with the appropriate adding power. The LCoS is then programmed to display a series of lenses to compensate for the disorder induced in the artificial eye. A fine-tuning of the optical power (or, equivalently, the focal length) of the programmable diffractive lens allows the compensation of the ametropia. We compare the compensation achieved by the programmable lens displayed on the LCoS with the compensation achieved by introducing a conventional ophthalmic lens from a trial lens case. To this end, we compute the modulation transfer function (MTF) for the compensation.

In a second stage, we used the SLM like a multifocal lens. We have examined the combination of diffractive lenses in one device [3,7]. In this stage we introduce four coding methods for merging the phase distributions of three Fresnel lenses, $\left(f_{1}, f_{2}, f_{3}\right)$, of different focal length into a single-phase distribution. The resulting phase is implemented experimentally via an LCoS SLM (HEO 1080P Holoeye) previously calibrated and characterized [8]. The combination of lenses is arranged for two configurations: coaxial and multiaxis. In the first one, all lenses have a common optical axis, whereas in the latter the lenses have parallel separated axes. We describe the main features of such lenses by taking into account the actual technical constraints given by the SLM specifications. We describe different methods for spatial encoding the three combined lenses, and we show numerical simulations and experimental results with an extended test-object for each coding method.

Finally, we study a several diffractive optical elements (DOEs) with EDOF. The most promising optical elements for imaging with EDOF in real-time seem to be optical elements focusing an incident plane wave into a focal line segment. These elements can be regarded as modified lenses with controlled aberrations. The modification should lead to output images characterized by the possible highest contrast, brightness and sharpness. Among the most important of these elements we present the Axicon [9], the Axilens (AXL)[10], the Light Sword Optical Element (LSOE) [11], and the Peacock Eye (PE) [4, 12]. Moreover, there are works that have shown the advantages of these optical elements as an alternative solution to the compensation of ophthalmic aberrations in aged human vision [4]. These elements illustrate a potential applicability of EDOF imaging components to presbyopia compensation. The PDOEs will be displayed on a parallel-aligned liquid crystal on silicon spatial light modulator (LCoS SLM), which works in phase only modulation regime [8]. The results obtained with all PDOEs will be compared with a multifocal lens. The latter consists of three phase diffractive lenses with the same axis that are spatially multiplexed. It has three focal points coinciding with the extremes and the center of the required depth of focus segment [7]. To test the optical performance of all the elements, we obtain the point-spread function (PSF), the modulation transfer function (MTF), and their evolution along the optical axis. To better visualize the 
imaging performance of each PDOE we acquire images of an extended object with incoherent illumination. The MTF is to be computed using a slanted border extracted from the image of the extended object. Additional results concerning incoherent imaging of extended objects placed at different distances are included. The results have been obtained experimentally.

\section{Representation of the PDOEs by means a pixelated device}

Let us denote the quadratic phase pattern of a spherical lens, in spatial coordinate $\left(x_{0}\right)$ and with focal length $f$, by $l\left(x_{0}, f\right)$, which is the continuous function

$$
l\left(x_{0}, f\right)=\exp \left(-j \frac{\pi}{\lambda f} x_{0}^{2}\right)
$$

represented in one spatial dimension for the sake of simplicity. The wavelength of the design is represented by $\lambda$. The pixelated structure of the SLM is responsible for a spatial discretization of the phase distribution displayed on the modulator. This discrete distribution can be written as

$$
l(m, f)=\exp \left(-\frac{j \pi}{\lambda f} \frac{L^{2}}{N_{l}^{2}} m^{2}\right) \quad \forall m \in\left[-\frac{N_{l}}{2}+1, \frac{N_{l}}{2}\right],
$$

where $m$ is the pixel position and $L$ is the diameter of the lens aperture that corresponds to a given number of pixels $N_{l}$. In general, $L \leq L_{0}$, where $L_{0}$ is the total SLM aperture. As Eq. (2) reveals, the phase value varies more rapidly in the periphery than in the center of the lens aperture (Fig. 1). According to the Nyquist criterion, the phase shift between two neighbor pixels of Eq. (2) at the border of the lens aperture must be lower than or equal to $\pi[13,14]$. This condition results in a minimum value for the focal length, named the reference focal length, of the programmable lens. For a given SLM, with a particular pixel pitch, the requirement establishes that the sampling frequency fits for the Nyquist criterion at the borders of the lens aperture. Assuming that $L=N_{l} \delta x_{0}$, where $\delta x_{0}$ is the pixel pitch, the reference focal length $f_{\text {ref, }}$ or equivalently, the reference power, can be determined from the expression $[13,14]$,

$$
f_{\text {ref }}=\frac{N_{l}}{\lambda} \delta x_{0}^{2}
$$

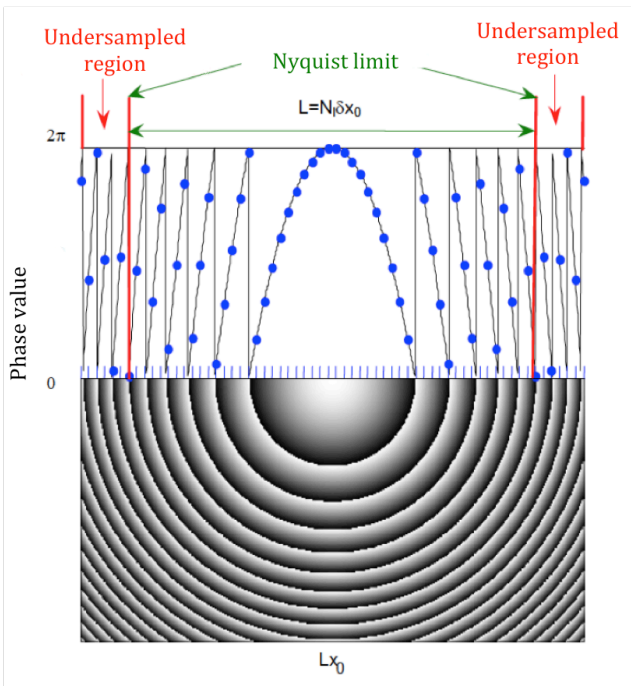

Fig.1. Spatial discretization and phase profile of a (modulo-2 $\pi$ ) programmable lens addressed to an SLM. Red dots represent the phase values addressed to the elements of the pixelated screen after the spatial discretization and phase quantization of the continuous phase profile (in solid blue line). Vertical red lines indicate the Nyquist limit beyond which the effects of undersampling may appear.

For instance, taking into account the specifications of Holoeye LCos-SLM used in this word $N_{l}=1080, \delta x_{0}=8$ $\mu \mathrm{m}$ ) and $\lambda=633 \mathrm{~nm}$, we obtain a $f_{r e f},=0.109 \mathrm{~m}$ or, equivalently, an optical power of 9.16 D. We assume that the SLM works in phase-only regime, with a minimal amplitude modulation and a linear phase response. We also assume that the phase modulation range is restricted to $2 \pi$ radians (modulo- $2 \pi$ ), which corresponds to the phase modulation depth available to reproduce the programmed phase lens with, for instance, a focal length of $f=f_{\text {ref. }}$. Furthermore, the phase modulation range is usually discretized into a 
number of levels addressable through the video graphic array card. In our experiments, 256 discrete phase levels were available.

\section{Compensation of refractive errors induced in an artificial eye through monofocals programmable lenses.}

Let us recall the principles for the compensation of refractive errors of ametropic human eyes. We firstly consider an artificial emmetropic eye (Fig. 2(a)) consisting of a camera lens, namely a photographic objective ( $L_{\mathrm{ob}}$ in Fig. 2), and a CCD sensor. For the proof of concept described in this work, we consider an over simplified version of the eye, hereafter named artificial eye. The photographic objective simulates the optical system of the eye whereas the CCD sensor plays the role of the retina. The relative position of these two elements is fixed, so that an object placed at a far distance, more specifically infinity, is focused on the CCD sensor placed at the back focal plane of the objective, $F_{\text {Lob }}^{\prime}$ in the same way that an emmetropic eye would focus the image of such an object on the retina. Different refractive errors can be simulated using this artificial eye in an optical bench. Let us briefly describe the myopia and the hyperopia, the astigmatism and the presbyopia.

\section{3.a. Refractive Errors}

Myopia and hyperopia: A myopic eye has higher optical power than an emmetropic eye. Consequently, the image of a far object through a myopic eye focuses in front of the retina. As depicted in Figure 2(b), an additional converging lens, $L_{\mathrm{M}}$, before the emmetropic artificial eye permits to induce myopia to it. In this artificial eye with induced myopia, the CCD sensor would capture a blurred image. In order to compensate the artificial eye for the induced refractive error, a third lens can be placed in front of the simulated myopic eye. A diverging lens, $L_{C}$, would compensate for the myopic effect introduced by $L_{\mathrm{M}}$, provided that the virtual back focal plane of $L_{C}\left(F_{\mathrm{LC}}^{\prime}\right)$ coincides with the front focal plane of $L_{\mathrm{M}}\left(F_{\mathrm{LM}}\right)$, that is, $F_{\mathrm{LC}}^{\prime} \equiv F_{\mathrm{LM}}$, as shown in Fig. 2(c). Taking into account the distance $d$ between $L_{\mathrm{C}}$ and $L_{\mathrm{M}}$ and the power of $L_{\mathrm{M}}$, we compute the power of $L_{\mathrm{C}}$, or equivalently, its focal length, to obtain a focused final image at the CCD sensor plane (Fig. 2(c)). Thus,

$$
f_{L C}^{\prime}=f_{L M}+d
$$

where the coordinate origin of each distance is indicated in Fig. 2(c). Those distances in the direction of light propagation are positive, and negative otherwise.

A hyperopic eye has lower optical power than an emmetropic eye. Consequently, the image of a far object through a hyperopic eye focuses behind the retina. Figure 3 shows this case. Hyperopia is induced in the artificial emmetropic eye by the use of a diverging lens, $L_{\mathrm{H}}$. A converging lens, $L_{\mathrm{C}}$, compensates for the refractive error provided that planes $F_{\mathrm{LC}}{ }_{\mathrm{LC}}$ and $F_{\mathrm{LH}}$ coincide at the same position of the optical axis, that is, $F_{\mathrm{LC}}^{\prime} \equiv F_{\mathrm{LH}}$. If this condition is met, the final in-focus image will be obtained in the CCD sensor plane (Fig. $3(\mathrm{c}))$.

(a)

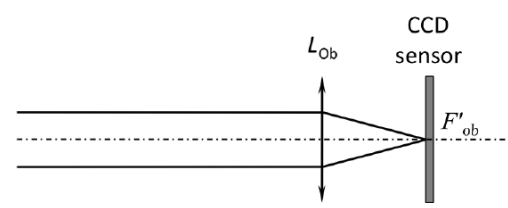

(c)

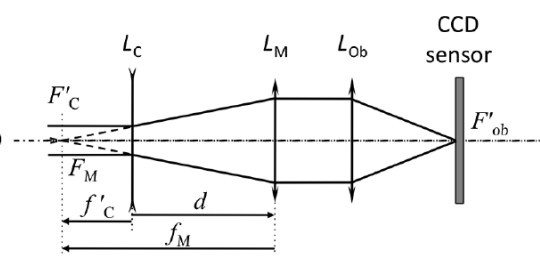

(b)

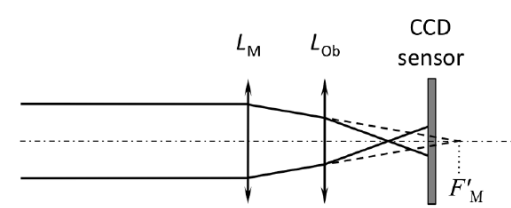

(d)

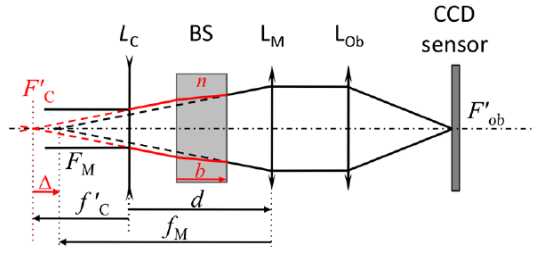

Fig. 2. Simulation of an artificial myopic eye on an optical bench and its ophthalmic compensation. (a) Emmetropic eye; (b) myopic eye (myopia induced by lens LM); (c) myopia compensation by lens LC; (d) longitudinal displacement, $\Delta$, introduced by the plano parallel plate effect of the beam splitter (BS).

Astigmatism: The introduction of a cylindrical lens $L_{A}$ in front of the artificial emmetropic eye permits to induce astigmatism. With the use of sphero-cylindrical or plano-cylindrical lenses in the position of $L_{M}$, a 
variety of cases can be simulated and described in terms of $L_{M}$ (Fig. 2) and $L_{H}$ (Fig. 3) for the principal meridians: from compound myopic astigmatism up to compound hypermetropic astigmatism, passing through simple myopic astigmatism, mixed astigmatism, and simple hypermetropic astigmatism. Applying in-plane rotations to the astigmatic lens $L_{A}$, we change the axis direction, or equivalently, the weaker and stronger principal meridians of the artificial astigmatic eye. As a consequence of the induced astigmatism, the CCD sensor captures blurred images. Although they appear to be different from the defocused images caused by simple spherical ametropia (i.e., myopia or hypermetropia), the compensation of the induced astigmatism does not involve, from the conceptual point of view, a rather different solution. The compensation with the lens $L_{\mathrm{C}}$ can be achieved by taking into separate consideration the two principal meridians of the artificial astigmatic eye. Apart from the blurring effects in the uncorrected astigmatic eye, there is some distortion in the image because of the different magnifications in the two principal meridians. After correction, a sharp image is restored, but, depending on the severity of astigmatism (i.e., the difference of power between the principal meridians) and the power and position of $L_{\mathrm{C}}$, some residual distortion may still affect the final image.

(a)

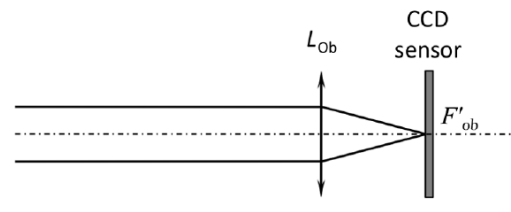

(c)

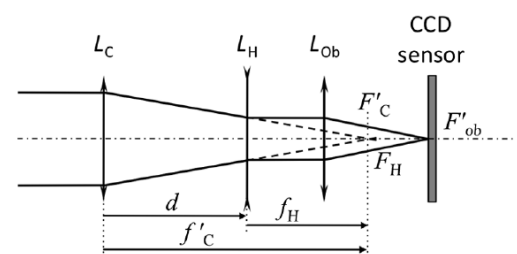

(b)

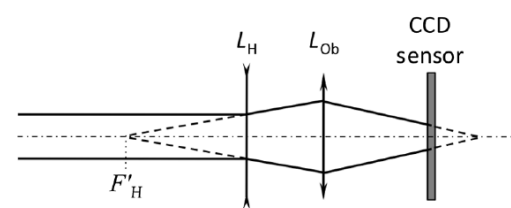

(d)

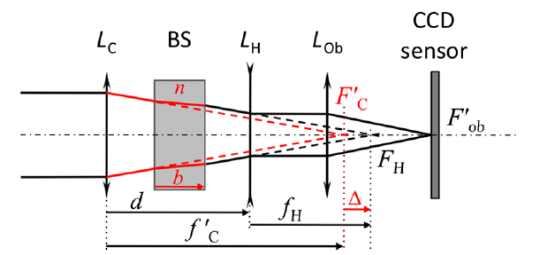

Fig.3. Simulation of an artificial hypermetropic eye on an optical bench and its ophthalmic compensation. (a)Emmetropic eye; (b) hypermetropic eye (hypermetropia induced by lens LH); (c) hypermetropia compensation by lens LC; and (d) longitudinal displacement, $\Delta$, introduced by the plano-parallel plate effect of the BS.

Presbyopia: The young eye is able to increase its power (accommodation) by modifying the curvature of the crystalline lens, and consequently, it is able of focusing on objects placed at different distances. Presbyopia is a decline in this focusing ability that appears as the crystalline lens ages. Therefore, when the emmetropic eye becomes additionally presbyopic, it needs an extra focusing power in the form of a "near addition" of positive power. If the presbyopic eye is either myopic or hypermetropic, it needs two kinds of compensations: to focus distant objects (distance correction) and to focus close objects (near addition). The near addition of positive power is added to the distance correction [15]. The need for a near addition depends on both the available amplitude of accommodation and the application demands (i.e., the working distance for the near visual task). It is possible to simulate this situation in an optical bench by placing the object test at a relatively close distance from the artificial eye (Fig. 4), for instance, about 10 times its focal length. A "young" emmetropic artificial eye would accommodate, that is, would increase its power (represented by the converging lens $L_{Q}$ in Fig. 4(a)) to focus correctly the object on the sensor. However, an "old" emmetropic eye that suffers from presbyopia has insufficient amplitude of accommodation, and the power increase (represented by the lens $L_{P}$ in Fig. 4(b)) is below demand. In such a situation, the presbyopic artificial eye forms the image behind the CCD sensor, thus capturing a blurred image of the object. This loss in the available amplitude of accommodation can be compensated by an additional converging lens, $L_{C}$, placed in front of the presbyopic artificial eye. This lens images the near object test, located at a distance $a$, onto the first focal plane of lens $L_{P}$, thus obtaining a sharp final image in the sensor plane [Fig. 4(c)]. The refractive power of the compensating lens $L_{C}$ must be adjusted so that the lens equation

$$
-\frac{1}{a}+\frac{1}{a^{\prime}}=\frac{1}{f_{C}^{\prime}},
$$

is fulfilled, and the condition for image distance $a^{\prime}=f_{P}+d$, is satisfied (Fig 4(c)). 

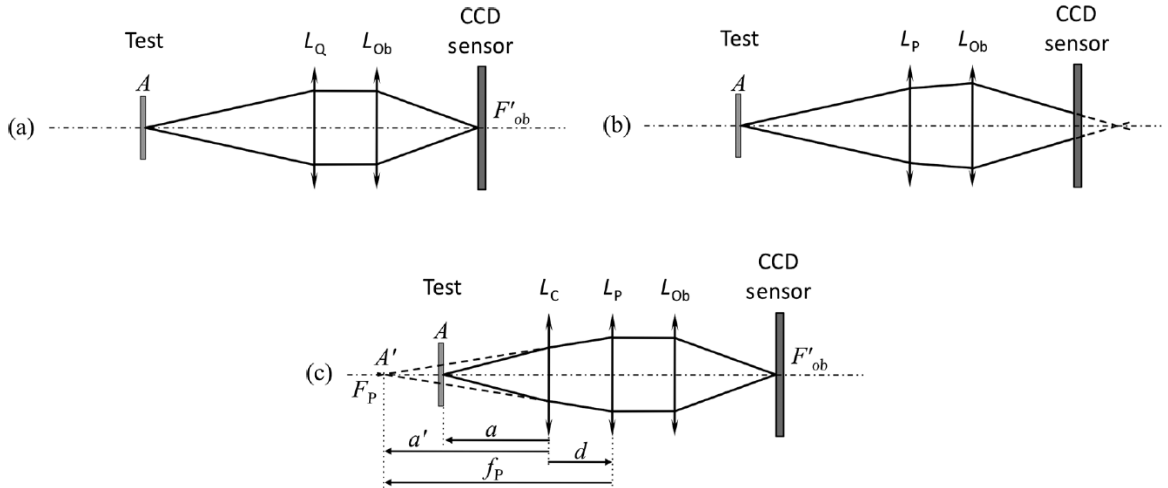

Fig. 4. Simulation of presbyopia on an optical bench and its ophthalmic compensation. (a) Accommodated eye with the object test placed at near distance. LQ provides the necessary additional optical power to the emmetropic eye (b) Presbyopic eye. The additional optical power of LP is under demand (c) Presbyopic eye compensated with the additional optical power of lens LC.

\section{3.b. Optical Experiments}

Experimental results are provided to compare the compensationachieved by programmable lenses displayed on an LCoS SLM with the compensation achieved by ophthalmic lenses from a trial-lens case. Figure 5 shows the setup used in the experiment. The object is illuminated with spatially incoherent light. The wavelength used is $\lambda=632.8 \mathrm{~nm}$ (He-Ne laser). The collimating lens $L_{1}$ permits to simulate that the object test is at a far distance (infinity) provided 0 is located in the front focal plane of lens $L_{1}$. This situation simulates the observation of a distance object by an emmetropic eye. We use the 1951 USAF resolution test chart as the object (Fig. 6). The SLM used to display the programmable compensating lens in our experiments, $L_{C}$, is an LCoS device that works in reflective mode. After reflection in the SLM, the BS reflects part of the modulated beam toward the artificial eye. The emmetropic artificial eye is represented in Fig. 5 by the combination of a lens (a photographic objective, $L_{o b}$ ) and a CCD sensor. Throughout the experiments, this artificial eye is going to be affected by myopia, hypermetropia, astigmatism, or presbyopia. The setup shown in Fig. 5 has been used to test the performance of the programmable lens displayed on the LCoS phase only panel as a compensating lens $L_{C}$ for refractive errors.

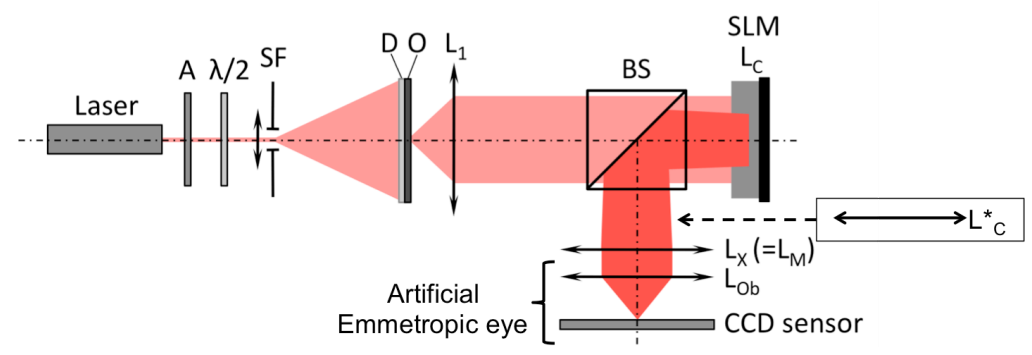

Fig. 5. Optical setup used in the experiment: A-attenuator, $\lambda / 2$-retarder half-wave plate, SF-microscope objective combined with a spatial filter, D-rotating diffuser, O-object test, L1-collimating lens, BS-beam splitter, SLM-spatial light modulator acting either as a programmable compensating lens $\left(L_{C}\right)$ or a flat mirror $(\mathrm{M})$, Lx-lens inducing the refractive error (being $\mathrm{X}=\mathrm{M}, \mathrm{H}, \mathrm{A}$, or $\mathrm{P}$ for a myopic, hypermetropic, astigmatic, or presbyopic eye, respectively), $L_{\mathrm{ob}}$-photographic objective and CCD sensor. When the trial lens was to be used for compensating the refractive error, $L^{*} \mathrm{C}$ was inserted closely before $L_{X}$ and, at the same time, the SLM was programmed to act as a flat mirror (M).

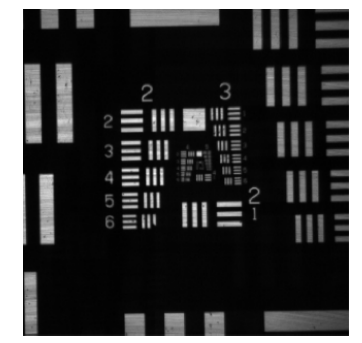

Fig.6. Experimental image of the standard 1951 USAF test chart captured by the CCD sensor of the artificial emmetropic eye. Only the central area of the image is displayed. White zones do not appear entirely uniform as an effect caused by the rotating diffuser placed against the test.

Experimentally we must consider that the compensating lenses $L_{C}$ and $L^{*} C$ would not have exactly the 
same power because of the different distances they are from the artificial eye. Besides, the presence of a BS in the ray path, between the SLM and the artificial eye, introduces a longitudinal displacement in the position of the second focal point of the compensating programmable lens $L_{C}$. The plane parallel plate equivalent to the right angle reflection prism of the BS produces such a displacement, which has to be taken into account in the refined calculation of the compensating power. The longitudinal shift $\Delta$ can be computed by the expression

$$
\Delta=\frac{n-1}{n} b,
$$

where $n$ and $b$ denote the refractive index and the cube dimension of the BS. For both cases, myopia and hypermetropia [Figs. 2(d) and 3(d)], this shift will modify the focal length of the programmed lens $f^{\prime} C$ by

$$
f_{C}^{\prime}=f_{x}+d-\Delta
$$

where $f_{X}$ will be $f_{M}$ or $f_{H}$ in case of myopia or hyperopia, respectively. Equation (7) is valid for compensating the astigmatism as well because it can be described in terms of $L_{M}$ and $L_{H}$ in the principal meridians separately.

Cylindrical ophthalmic lenses were successively placed in front of the emmetropic eye to simulate simple astigmatism in different directions ( $L_{X}=L_{A}$ in Fig. 5). Simple myopic astigmatism in the horizontal direction was induced by inserting a $+1.5 \mathrm{D}$ cylindrical lens (first focal length $f_{A}=-666.6 \mathrm{~mm}$ ), whereas simple hypermetropic astigmatism in the vertical direction was induced by inserting a $-1.5 \mathrm{D}$ cylindrical lens (first focal length $f_{A}=+666.6 \mathrm{~mm}$ ). Fig. 7(a) show the corresponding defocused images as the artificial astigmatic eye in each situation captured them. Ametropy compensation was first achieved by a programmed cylindrical lens displayed on the SLM with the same orientation as the ophthalmic cylindrical lens used as $L_{A}$. According to Eq. (7), and keeping $d=90 \mathrm{~mm}$, the calculated focal lengths to compensate for the horizontal and vertical ametropia were $f^{\prime}{ }_{C}=-594 \mathrm{~mm}$ and $f^{\prime}{ }_{C}=740 \mathrm{~mm}$, respectively. The focal length of the programmed lens displayed on the SLM was adjusted to experimentally optimize the compensation. The experimental focal lengths that best performed were $f^{\prime}{ }_{C}=-578 \mathrm{~mm}$ and $f^{\prime}{ }_{C}=+813$ $\mathrm{mm}$, respectively, which are in good agreement with the predicted values. Sharply focused images were obtained by the compensated artificial eye, and they can be seen in Fig. 7(b).

(a)
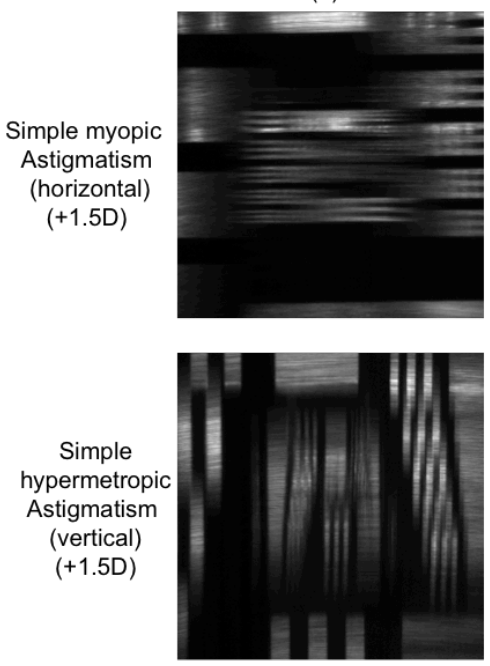

(b)
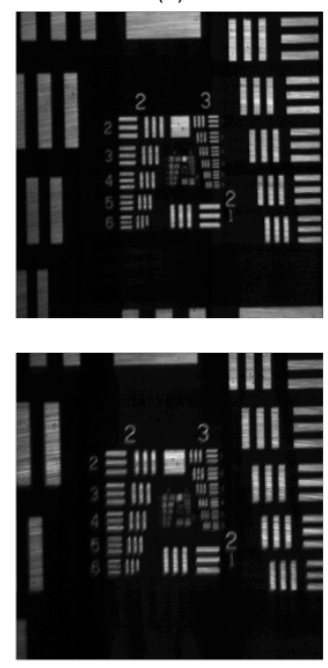

(c)
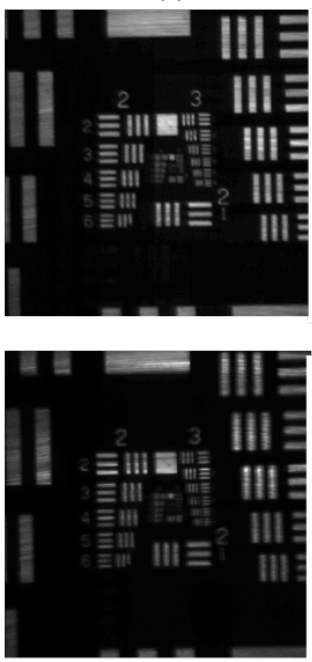

(d)
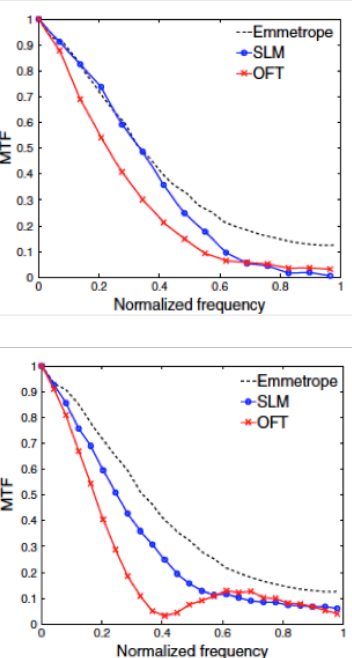

Fig. 7. Experimental results for the compensation of the induced simple myopic astigmatism in the horizontal direction (left), simple hypermetropic astigmatism in the vertical direction (right). (a) Defocused image due to the induced astigmatism; focused image obtained in the CCD sensor when the compensation was done by (b) display in a programmable lens on the SLM or, (c) using an ophthalmic cylindrical lens; (d) MTF functions computed from the focused images (b) and (c), along with the MTF of the emmetropic eye.

Conventional compensation by ophthalmic trial lenses placed in front of the artificial eye while addressing a flat mirror to the SLM was also carried out. The available cylindrical trial lens of $-1.5 \mathrm{D}$ optical power was used to compensate for the simple myopic astigmatism for a distance $d=10 \mathrm{~mm}$. For the 
compensation of the simple hypermetropic astigmatism, a cylindrical lens of $+1.5 \mathrm{D}$ from the trial set was used. Figure 7 (c) contains the captured images after the compensation for the simple myopic astigmatism in the horizontal direction (left) and the simple hypermetropic astigmatism in the vertical direction (right) by these conventional ophthalmic trial lenses. The quality of these images is compared with the images obtained after the compensation done by the programmed lenses on the SLM, through the computation of the MTF curves [Fig. 7(d)]. In both cases, the best performance corresponds to the programmable compensating lenses displayed on the SLM, whose MTF curves have a slower decrease in the frequency domain than the conventional trial lenses and are closer to the emmetropic behavior. In fact, the compensation of simple hypermetropic astigmatism (vertical) with a trial lens presents an MTF curve that reveals a pseudoresolution effect (side lobe centered at $\approx 0.65$ cycles/pixel) with reversal contrast, thus indicating the mediocre quality of this compensation.

To simulate presbyopia in the optical bench, a near object was obtained by approaching lens $L_{1}$ to the USAF test ( $O$ in Fig. 5 ) so that its conjugated image, which corresponds to test A in Fig. 4, was located approximately at $500 \mathrm{~mm}$ from the artificial eye. In such a situation, the acquired image by the emmetropic eye was defocused, unless sufficient accommodation (about +2 D) was available. We assumed the accommodation capability was limited to just $+1 \mathrm{D}$, that is, the artificial eye suffered from presbyopia. To simulate this presbyopia, a lens $L_{\mathrm{P}}$ of $+1 \mathrm{D}$ was placed at the location of $L_{\mathrm{X}}$ (Fig. 5) and a defocused image was captured as shown in Fig. 8(b). From the optical power of $L_{P}$ (or equivalently, its focal length

$f^{\prime}{ }_{\mathrm{P}}=1000 \mathrm{~mm}$ ) and taking into account that the distance between the compensating lens and the artificial presbyopic eye was $d=110 \mathrm{~mm}$, the necessary additional power of the compensating lens $L_{\mathrm{C}}$ was+1.44 D. Compensation for presbyopia was first attempted with the programmable lens displayed on the SLM. A range of focal lengths from +600 to $+720 \mathrm{~mm}$ in steps of $5 \mathrm{~mm}$ were sequentially addressed to the LCoS device, obtaining the best compensation for $f^{\prime}{ }_{C}=+700 \mathrm{~mm}$, which corresponds to an optical power of +1.43 D. The captured image after this compensation is shown in Fig. 8(c). After that, we applied the alternative compensation with a conventional trial lens. If a plane mirror was displayed on the SLM, and an ophthalmic trial lens of $+1.5 \mathrm{D}$ was used, the compensation was also achieved as shown in Fig. 8(d). Comparing both results in terms of MTF values [graphs depicted in Fig. 8(e)], a better performance of the programmable lens is obtained in comparison to the ophthalmic trial lens.

(a)

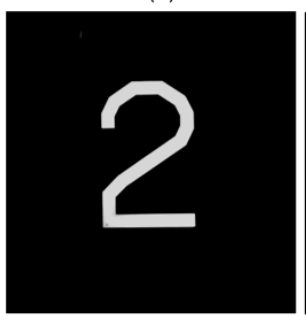

(b)

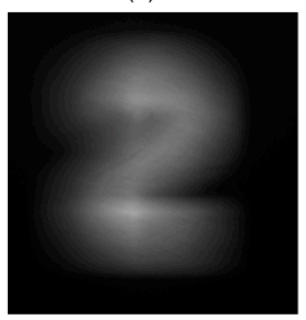

(c)

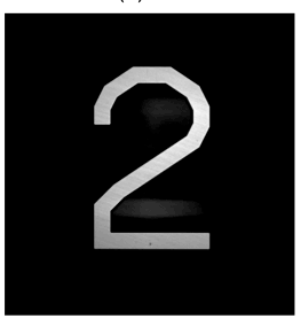

(d)

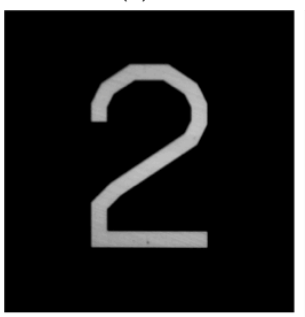

(e)

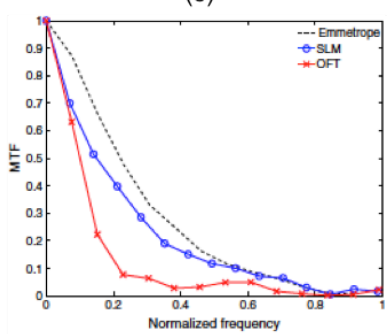

Fig. 8. Experimental results of the compensation for presbyopia. (a) Reference: captured image of a distant object by the artificial emmetropic eye. (b) Defocused image of a near object (placed at $500 \mathrm{~mm}$ from the eye approximately) obtained when the presbyopic artificial eye has just $+1 \mathrm{D}$ of accommodation. (c) Refocused image after compensation with the programmable lens displayed on the SLM, which provided the necessary additional power (focal length finely tuned to $+700 \mathrm{~mm}$ ). (d) Refocused image after compensation by an ophthalmic trial lens with additional optical power of +1.5 D. (e) MTF curves corresponding to figures (a), (c), and $(\mathrm{d})$.

\section{Optical implementation of multifocal programmable lens.}

\section{4.a. Spatial encoding the multifocal lens}

To combine multiple lenses in a single-phase distribution, two different encoding methods of the phase distribution are used: by rows or randomly. In the codification by rows, each row of pixels of the modulator is assigned to one sublens, and the process is repeated sequentially until filling in the SLM aperture. In the random codification, each pixel $(x, y)$ of the SLM's aperture is assigned a value of $\mathrm{k}$ between 0 and 1 , randomly. If $k \leq(1 / 3)$, the pixel represents the phase of $l_{1}(x, y)$. If $(1 / 3)<k \leq(2 / 3)$, the pixel represents the phase of $l_{2}(x, y)$ and finally, if $(2 / 3)<k$ the pixel represents the phase of $l_{3}(x, y)$.

Two configurations of multifocal lens are considered: the lenses share a common optical axis (coaxial configuration) or have different parallel axes (multi-axis configuration). In all cases, three sublenses $l_{1}, l_{2}$, $l_{3}$ with respective focal lengths $f_{1}, f_{2}, f_{3}$, are combined. All the focal lengths used in these experiments are greater than $f_{\text {ref. }}$ The focal lengths for $l_{1}, l_{2}, l_{3}$ sublenses are $f_{1}=35 \mathrm{~cm}, f_{2}=25 \mathrm{~cm}$ and $f_{3}=30 \mathrm{~cm}$, respectively, 
where $f_{2}$ is the closest to the $f_{\text {ref }}$ of the Eq. 3

Coaxial configuration. The optical axes of the three sublenses $l_{1}, l_{2}, l_{3}$ coincide. Figure 9 shows a grey level representation of the phase distribution of the multifocal lens with coaxial configuration: (a) with phase codification by rows, (c) with random codification of phase.

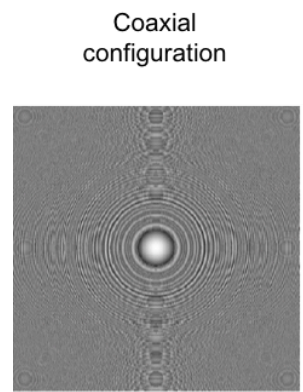

(a)

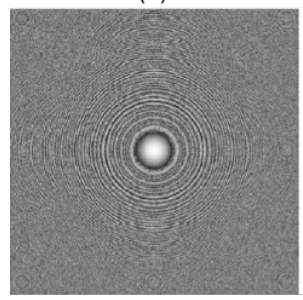

(c)

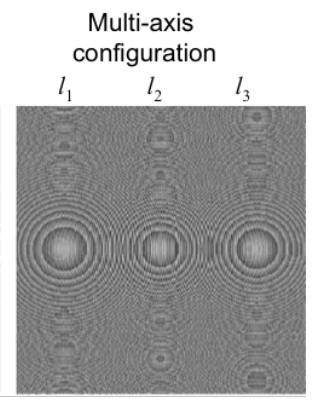

(b)

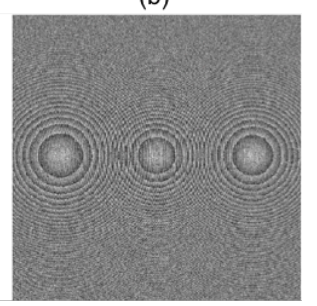

(d)

Fig.9. Phase distributions for three lenses $\left(l_{1}, l_{2}, l_{3}\right)$ of different focal length in coaxial and multiaxis configuration. (a) and (b) Multifocal lens coded by rows, (c) and (d) multifocal lens randomly coded.

Multi-axis configuration. In this configuration the optical axes of the three sublenses are separate and parallel between them. Each lens can be described as follows: $l_{1}\left(x_{0}+x_{d}, f_{1}\right), l_{2}\left(x_{0}, f_{2}\right), l_{3}\left(x_{0}-x_{d}, f_{3}\right)$. Figure 9 shows a grey level representation of the phase distribution of the multifocal lens with multi-axis configuration: (b) with phase codification by rows, (d) with random codification of phase.

\section{4.b. Simulated and Experimental results of the multifocal lens}

In order to obtain the images formed by programmable multifocal lens designed according to the different configuration shown in section 4.a, we present the numerical simulation results obtained by means of Fresnel propagation. The experimental results were obtained using the setup of the figure 5 , but for this experiment, the CCD camera operated without photographic objective, that is, the lenses $L_{\mathrm{X}}$ and $L_{\mathrm{ob}}$ were removed. In this way, the multifocal lens formed images at different distances in the image space. Displacing conveniently the CCD sensor the images were captured.

In Fig. 10 we show the simulated and experimental results obtained in the image plane for the multi-focal lens coded by rows and with coaxial configuration. In the coaxial configuration the three images overlap due to the fact that the optical axes of the three sublenses coincide. When the three sublenses are regularly encoded by rows on the SLM, vertical repetitions of the image appear due to the multiple diffraction orders introduced by the coding procedure. We verified the correspondence between the numerical and experimental results, although, only the central zero-order image is shown because the images of the higher orders were outside the field of the CCD.

In Fig. 11 we show the simulated (above) and experimental (below) results obtained in the image plane, for the multi-focal lens with coaxial configuration and random phase encoding. In each image plane a coaxial superposition of three images is observed, while one of them is in focus the other two appear defocused.

Figure 12 shows the results for the multifocal lens with multi-axis configuration and random phase encoding. In this case, the multi-axis configuration does not form a mixture of in-focus and out-of-focus superposed images. The multi-axis configuration permits to spatially separate these images. 


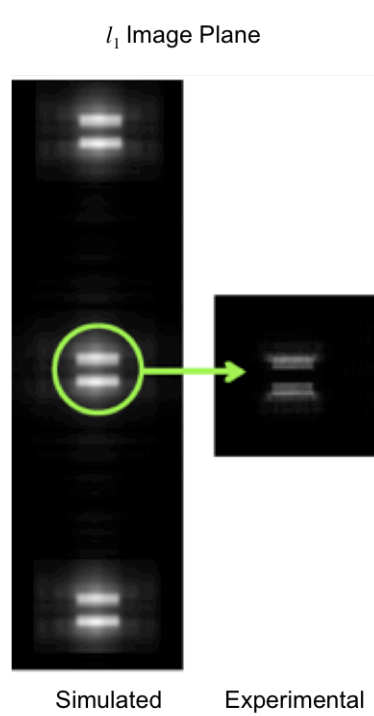

(a)

(b)

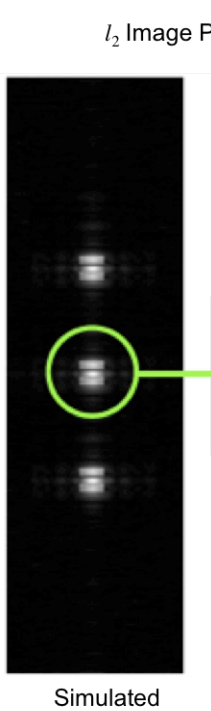

(c)

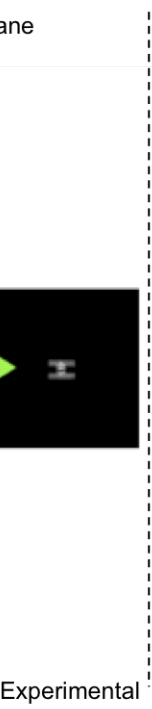

(d)

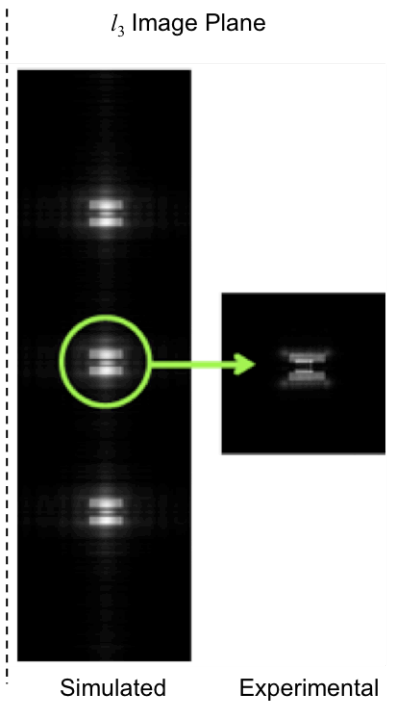

(e)

(f)

Fig. 10. Coaxial configuration coded by rows. The images (a), (c), (e) represent simulated image planes and (b), (d), y (f) experimental results. (a)-(b) $l_{1}\left(f_{1}=35 \mathrm{~cm}\right)$, (c)-(d) $l_{2}\left(f_{2}=25 \mathrm{~cm}\right)$, (e)-(f), $l_{3}\left(f_{3}=30 \mathrm{~cm}\right)$.
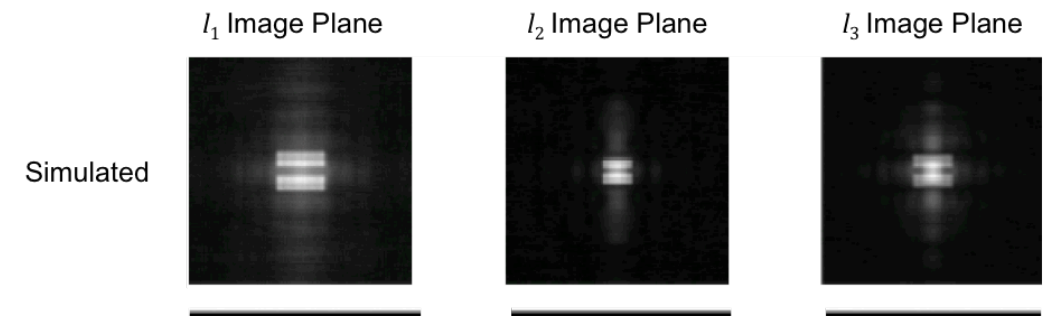

Experimental
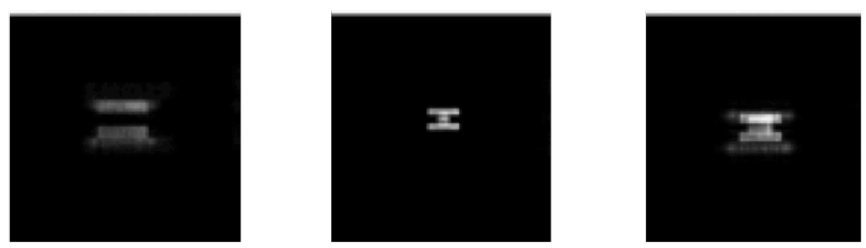

Fig. 11. Multifocal lens with coaxial configuration and random phase encoding. Simulated (top) and experimental (bottom) results. $11(\mathrm{f} 1=35 \mathrm{~cm}),(\mathrm{c})-(\mathrm{d}) 12(\mathrm{f} 2=25 \mathrm{~cm}),(\mathrm{e})-(\mathrm{f}), 13(\mathrm{f} 3=30 \mathrm{~cm})$
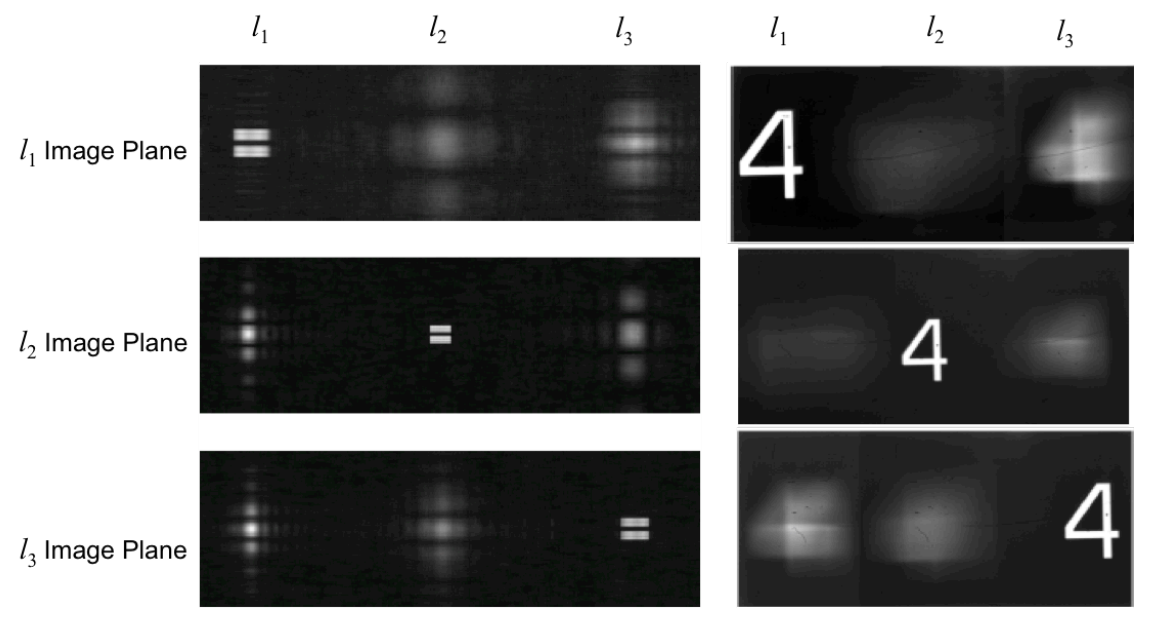

Fig. 12. Multifocal lens with multi-axis configuration and random phase encoding. Simulated (left) and experimental (right) results. $11(\mathrm{f} 1=35 \mathrm{~cm}),(\mathrm{c})-(\mathrm{d}) 12(\mathrm{f} 2=25 \mathrm{~cm}),(\mathrm{e})-(\mathrm{f}), 13(\mathrm{f} 3=30 \mathrm{~cm})$.

We found the experimental results to be consistent with the numerical simulations carried out for each configuration. The experimental results bring out the fact that the multi-axis configuration is 
characterized by providing a good contrast in the images planes that are in focus. On the other hand, the coaxial configuration images with poor contrast due to the image overlapping on the same axis. Regarding the encoding methods, we have verified that the row encoding method generates multiorder image replicas given by the repeating pattern introduced by the codification, whereas the random encoding method has not this disadvantageous effect.

\section{Programmable Diffractive Optical Elements for Extending the Depth of Focus}

In this section we evaluate an optical performance of a variety of Proframmable DOEs with EDOF such as: the Forward Logarithmic Axicon (FLAX) [9], the Axilens (AXL) [10], the Light Sword Optical Element (LSOE) [11], the Peacock Eye Optical Element (PE)[16] and Double Peacock Eye Optical Element (DPE) [4]. These elements illustrate a potential applicability as EDOF imaging components for presbyopia compensation. The PDOEs will be displayed on a LCoS SLM. The results obtained with all PDOEs will be compared with a multifocal lens. The latter consists of three phase diffractive lenses with the same axis (coaxial configuration) that are spatially multiplexed. It has three focal points coinciding with the extremes and the center of the required depth of focus segment. To test the optical performance of all the elements, we obtain the point-spread function (PSF), the modulation transfer function (MTF), and their through-focus evolution along the optical axis. To better visualize the imaging performance of each PDOE we show images of an extended object with incoherent illumination. The MTF was computed using a slanted border extracted from the image of the extended object. The results shown have been obtained experimentally.

\section{5.a. Programmable DOEs}

Forward logarithmic axicon optical element (FLAX): An axicon is an optical element that transforms an incident plane wave into a narrow focal segment with uniform intensity. It is characterized by a refractive power that decreases with the radial distance $(r)$ to its center, thus the peripheral rays are focused to an axial point located farther than the focus for the central rays. Figure 13(a) shows the geometrical parameters and distribution of rays for an axicon illuminated by a plane wave of wavelength $\lambda$. The transmittance function for this element has the following form:

$$
t(r)=\left[-i k / 2 a \ln \left(1+a r^{2} / f_{1}\right)\right] ;
$$

where $k=2 \pi / \lambda$ and $a=\left(f_{1}-f_{2}\right) / R^{2}=\Delta f / R^{2}$ and $r=\left(x^{2}+y^{2}\right)^{1 / 2}$. Figure 14(a) shows the resulting phase distribution of this DOE represented in grey levels. This is what is ultimately sent to the modulator.

Axilens optical elements ( $A X L$ ): This element also concentrates the incoming energy in a segment of the optical axis. It has a focal length that varies with the radial coordinate but the associated phase retardation function differs from the conventional quadratic phase. This element is composed of concentric rings of infinitesimal width. $R$ is the radius of the lens aperture, $f$ and $\Delta f$ represents the focal of the lens and the length of the focal segment respectively. Figure 13(b) shows the geometrical parameters and distribution of rays for an axilens illuminated by a plane wave of wavelength $\lambda$ and Figure 14(b) shows the resulting phase distribution of this DOE represented in grey levels. The transmittance function of the axilens can be written $[10]$ as

$$
t(r)=\left\{-i k r^{2} / 2\left[f_{1}+\left(\Delta f r^{2} / R^{2}\right)\right]\right\} ;
$$

Light Sword Optical Element (LSOE) This element is a counterpart of the axilens where the radial modulation of the focal has been replaced by an angular one [11]. The transmittance function has the following form in the polar coordinate system:

$$
t(r, \theta)=\left\{-i k r^{2} / 2\left[f_{1}+(\Delta f \theta / 2 \pi)\right]\right\} ;
$$

where $r, \theta$ are the radial and angular coordinates respectively. Analyzing Eq. (10), we can see that the phase function of the LSOE is an unconventional Fresnel lens that has a focal length $f+\Delta f / 2 \pi$. Therefore, the LSOE focuses an incident plane wave into a focal segment $\Delta f$. When $\theta \in[0,2 \pi)$ then the segment is stretched from a distance $f_{1}$ to a distance $f_{1}+\Delta f$ in front of the LSOE plane. This segment is oriented perpendicularly to the sector, like is shown in Fig.13(c). Figure 14(c) shows the resulting phase distribution of this DOE represented in grey levels. This is what is ultimately sent to the modulator 
Peacock eye optical element (PE) [4]: The geometrical parameters and distribution of rays for a peacock eye and illuminated by a plane wave of wavelength $\lambda$ are shown in figure 13(d). In the PE element, the focal segment is aligned with the optical axis. The transmittance function of this PDOE by which an incident plane wave is focused onto a focal segment of the optical axis with uniform intensity distribution is given by

$$
t(x, y)=\left[\frac{-i k y^{2}}{2(L x / A+d)}-\frac{A}{L} x+\frac{d A^{2}}{L^{2}} \ln \left|\frac{L}{A} x+d\right|\right]
$$

where $A$ is a square aperture uniformly illuminated by a plane wave of wavelength $\lambda$. L is the length of the focal segment of extremes $\left[f_{1}, f_{2}\right]$. The central point of this segment is at a distance $d$ from de PE aperture. Figure 14(d) shows the resulting phase distribution of this DOE represented in grey levels.

Double Peacock eye (DPE): The Double peacock eye element is made by spatially multiplexing two single PE elements [4] These single PEs elements were designed so that their corresponding focal segments are arranged in such a way that one focal segment is located after the other along the optical axis, with some partial overlapping. The total length covers the required depth of focus. We have considered a Random distribution configuration for the design of the double multiplexed peacock eye. In this configuration the device aperture is segmented into small windows of $3 \times 3$ pixels. The phase of either one or the other peacock eye (only one of them) is displayed on each window according to a mosaic random distribution (Figure 14(e)).

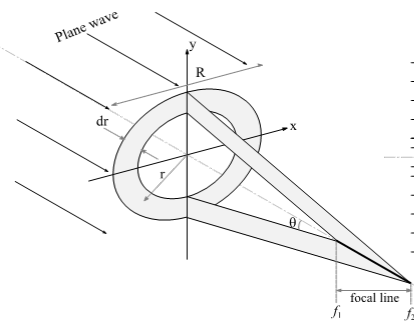

(a)

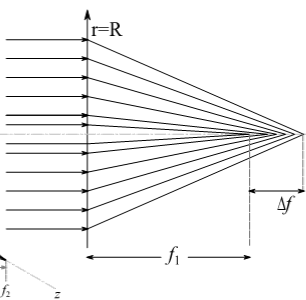

(b)

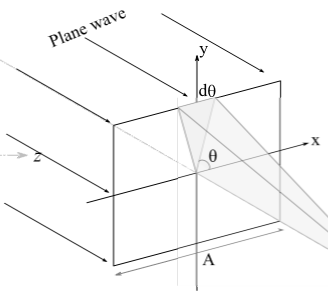

(c)

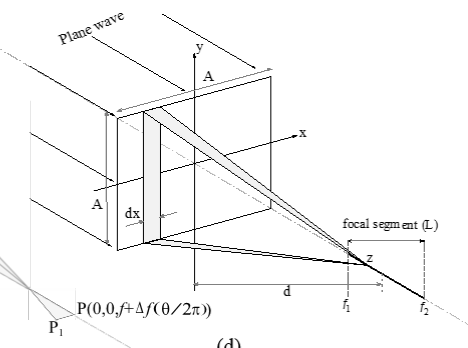

(d)

Fig. 13. Geometrical parameters and schematic distribution of rays with an input plane wave focused by all DOEs. (a) FLAX-(b) AXL, (c) LSOE, the infinitesimal angular sector of the element focuses an incident plane wave onto a segment PP1 oriented perpendicularly to the optical axis, (d) PE.

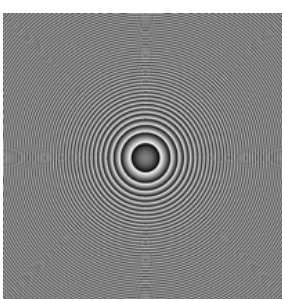

(a)

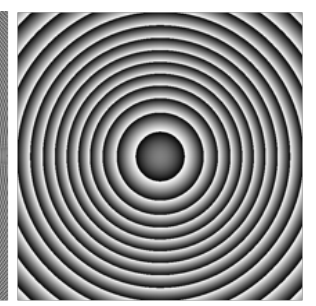

(b)

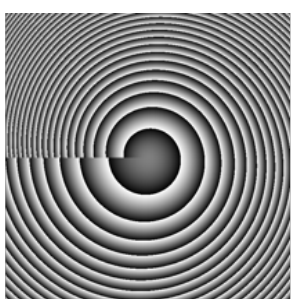

(c)

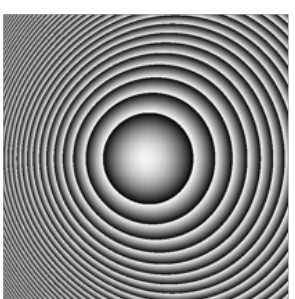

(d)

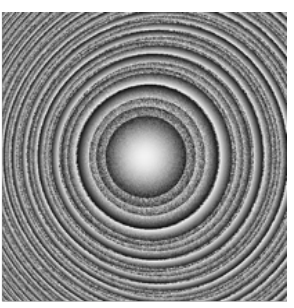

(e)

Fig.14. Phase distributions for the PDOEs in represented in grey levels. (a) FLAX, (b) AXL, (c) LSOE, (d) PE, (e) DPE.

\section{5.b. Optical experiment and programmable DOEs design conditions}

All programmable DOEs were designed so that the DOF lies within a specific range of focals giving rise to a focal segment within the interval $\left[f_{1}, f_{2}\right]$ with fixed extremes at the axial distances of $f_{1}=30 \mathrm{~cm}$ (power in diopters of $3.33 \mathrm{D}$ ) and $f_{2}=80 \mathrm{~cm}(1.25 \mathrm{D})$. In the case of the single PE had a focal segment $\left[f_{1}, f_{2}\right]$ that coincided with the requested focal segment, that is $f_{1}=30 \mathrm{~cm}$ and $f_{2}=80 \mathrm{~cm}$. The double PE had two focal segments $\left[f_{1}, f_{2}\right]$, and $\left[f_{3}, f_{4}\right]$, that covered the requested total focal segment, with some overlap (of about $5 \mathrm{~cm}$ ) in the center, that is, $f_{1}=30 \mathrm{~cm}, f_{2}=58 \mathrm{~cm}, f_{3}=53 \mathrm{~cm}$ and $f_{4}=80 \mathrm{~cm}$. In the optical experiment (Figure 13), each phase diffractive element was displayed on the SLM controlled by computer. A He-Ne laser beam (element 1 in Fig. (15)) was used for illumination. Using a small pinhole (element 4) in the optical axis, the beam was spatially filtered and afterwards collimated to obtain the PSFs produced by each optical element displayed on the SLM (element 9) in the first series of experiments. In the second series of experiments, a ground glass rotating diffuser (element 5) was located against the object (element 6) to obtain incoherent illumination. The extended object was placed at the front focal distance of an auxiliary lens of $f_{\text {aux }}=200 \mathrm{~mm}$, thus the image is located at infinity. The extended object used is the figure \#2 from 
the test USAF $\left(1.5 \mathrm{~mm}\right.$ lateral size) and it covered an angular field $\approx 0.07^{\prime} \approx 4.2$ ". The object was imaged by the DOE displayed on the SLM. A CCD sensor (element 11) was displaced through several planes of the focal segment for capturing the image. We fixed the capturing parameters of the CCD camera so as to avoid the saturation of the camera.

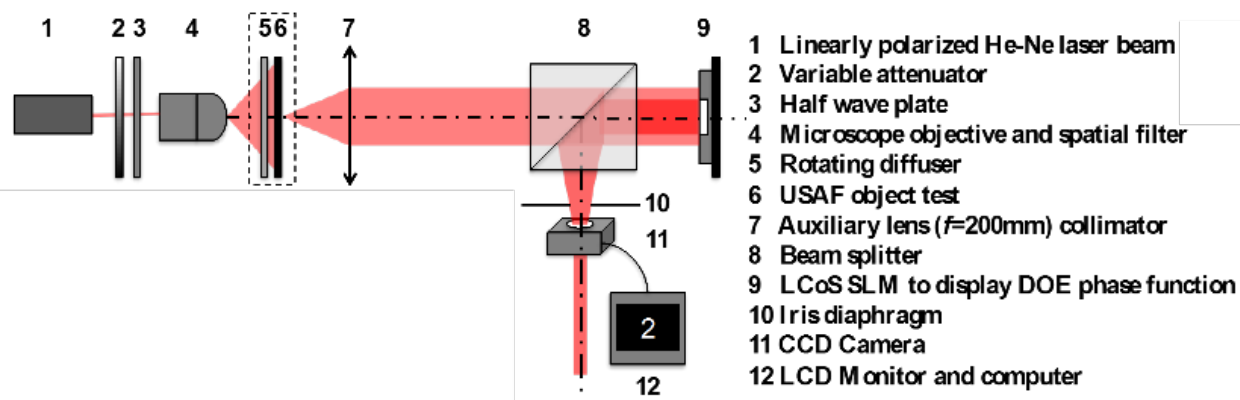

Fig.15. Experimental setup to evaluate the extended depth of focus of PDOEs. This setup is used to image a diffusing extended object (element 6) in the second series of experiments. To obtaind the PSFs (first series of experiments) the element 5 and 6 are removed and the element 4 is shift to their position.
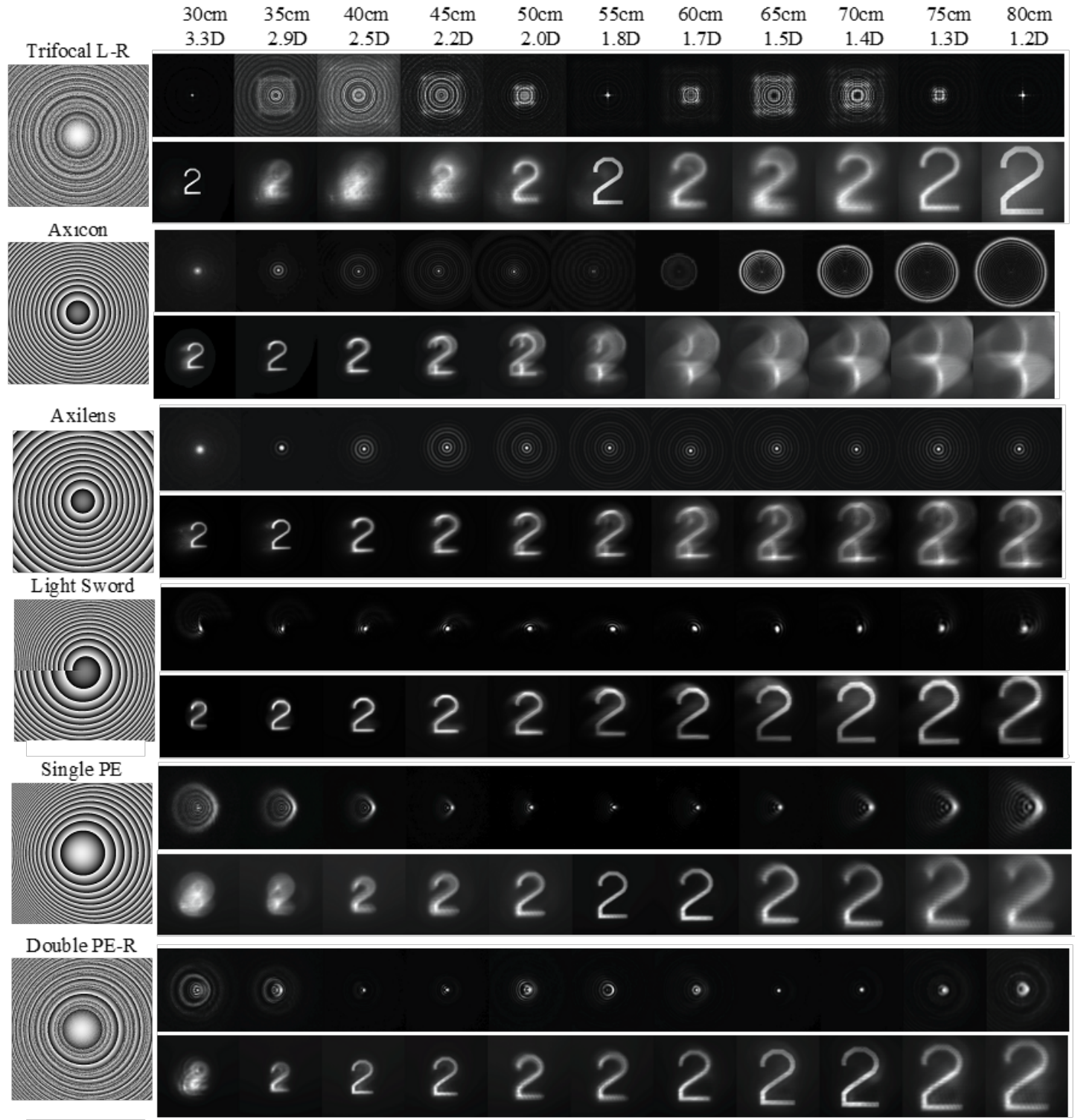

Figure 16. Experimental PSFs and images of an extended object along different positions of the focal segment obtained with the phase of the PDOEs represented in gray levels on the left column. The positions from the PDOE (top) are expressed in cm and diopters (D). 
Figure 16 shows the experimental results for the PSFs and the images of the extended object along different positions of the focal segment obtained for the different PDOEs. Figure 17 shows experimental MTFs calculated from the PSFs of the PDOEs (Fig 17). The spatial frequency of the MTFs is normalized for the all cases. As for the constant of normalization, we have considered the diffraction-limited cutoff spatial frequency in the object space, in our experiment $f_{\text {cutoff }}=238$ cycles/degree. For the some DOEs, we present two cross-sections of the MTFs corresponding to perpendicular directions, horizontal (H) and vertical (V).

An overview of the image quality produced by all DOEs along the focal segment reveals that with the exception of the multifocal lens, all remaining elements extend the DOF. However, not all elements preserve image quality significantly along the focal segment. In both the Axicon and Axilens, their PSFs collect most of the incident energy in the central maximum. However, the energy collected around external rings is not negligible, which leads to a decrease in image quality. Because the central maximum concentrates most of the energy along several planes of the focal segment, the DOF is extended. In the case of the FLAX it performs sufficiently well in the first half of the focus range and the performance decreases for the following planes, although it is not a considerable decline. The best image quality appears in the planes of the focal segment, roughly from $30 \mathrm{~cm}$ to $50 \mathrm{~cm}$. In the case of the AXL the best image quality appears in the planes of the focal segment from $40 \mathrm{~cm}$ to $80 \mathrm{~cm}$. Analyzing the MTF of the axicon we can see that for distances greater than $z=50 \mathrm{~cm}$ the MTFs decrease rapidly from the zeroth frequency onward. There are contrast inversions that produce blurry images. In contrast to the effect observed in the MTFs of the axicon, the MTFs of the axilens do not decrease as rapidly and have greater resemblance between them. Which means that it is more insensitive to misfocus than the axicon. This is evidenced by the images of the extended object, which is better defined throughout most of the focal range $\Delta \mathrm{z}$.

The LSOE collect a high amount of incident light at the most intense part of the maximum. These elements have the property of focusing within a focal segment, however the main maximum is not located on the optical axis. From the experimental results, we have located and plotted a series of maxima along the focal segment for different image planes for both elements. In the case of the LSOE it performs sufficiently well in the intermediate image planes and the performance decreases at the extremes $\left(f_{1}=30 \mathrm{~cm}\right.$ and $\left.f_{2}=80 \mathrm{~cm}\right)$, although it is not a considerable decline. The best image quality appears in the planes near the central part of the focal segment, roughly from $50 \mathrm{~cm}$ to $70 \mathrm{~cm}$. In the focal planes outside this range, the images degrade relatively quickly. These results are consistent with the MTFs shown in Figure 17.

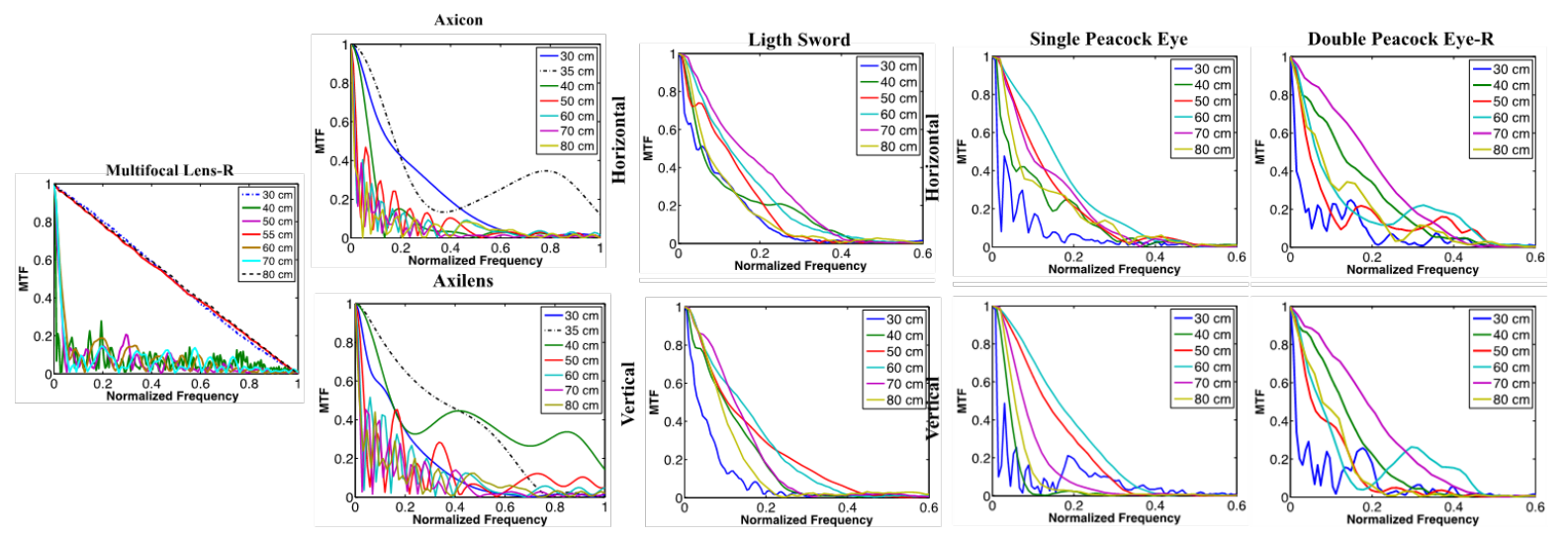

Figure 17. Experimental MTFs computed from the PSFs of Figure 16

The performances of the peacock-based elements show a real focal segment, somewhat shorter than expected, where defocus is remarkably reduced. In the case of the single peacock eye, the best image quality appears in the central part of the focal segment, let us say, from $50 \mathrm{~cm}$ up to $60 \mathrm{~cm}$. Images degrade rather quickly outside this central part toward the extremes of the designed focal segment (from $30 \mathrm{~cm}$ to $80 \mathrm{~cm}$ ). In case of the double peacock eyes, however, the image quality benefits from two separate segments of good performance (the first, from $35 \mathrm{~cm}$ up to $45 \mathrm{~cm}$ and the second, from $65 \mathrm{~cm}$ to $75 \mathrm{~cm}$ ), yet maintaining an acceptable performance in the central part (from $50 \mathrm{~cm}$ up to $60 \mathrm{~cm}$ ) of the total focal segment, where both focal components overlap. Even in the extremes of the total focal. These results shed some light on the problem of designing optical systems with defocus invariance. Moreover, there exists a potential applicability for ophthalmic applications, like in presbyopia compensation. 


\section{5.c. Double peacock eye optical element for extend depth imaging for presbyopia compensation}

We illustrate the potential applicability of the phase peacock-based diffractive elements as EDOF imaging components for presbyopia compensation. The object was axially shifted from the infinite (object vergence in diopters equal to $0 \mathrm{D}$ ) toward the LCoS SLM. To cover long object distances (from infinite (0D) to $1 \mathrm{~m}$ (1D), approximately), we shifted the real object within the front focal distance of the auxiliary lens $\left(f_{\text {aux }}=20 \mathrm{~cm}\right)$. For shorter object distances, we removed the auxiliary lens and directly shifted the object along the bench toward the LCoS SLM. Figure 18 shows the experimental images captured by the camera for the two elements: the single peacock eye and the double peacock eye. The latter was multiplexed based on the addition of transmittances. At short and intermediate object distances (up to $165 \mathrm{~cm}$ or object vergence $0.61 \mathrm{D}$ ), Figure 18 clearly demonstrates better results for the DPE than for the single PE. For far object distances (from $165 \mathrm{~cm}$ to infinite), however, the single peacock eye assures more accurate results.

In the case of the double peacock eye, we should say that the image obtained for the object placed at $90 \mathrm{~cm}$ is still acceptable in comparison with the others; consequently, this position constitutes the "near object point" for the EDOF imaging element. Since the image plane of the near object point is located at $65 \mathrm{~cm}$ (image vergence $1.5 \mathrm{D}$ ) behind the LCoS SLM, it implies that the double peacock eye is operating with a focal length of $38 \mathrm{~cm}$ (power of $2.6 \mathrm{D}$ ) approximately according to a simple calculation in the paraxial optics approach. This result is consistent with the values considered in the design of this PDOE (a total focal segment from $f_{1}=30 \mathrm{~cm}$ to $f_{2}=80 \mathrm{~cm}$ ). The "near object point" for the single peacock eye would be at $142 \mathrm{~cm}$ (object vergence $0.70 \mathrm{D}$ ) approximately. The "remote object point" would be infinite for the single peacock eye, whereas for the double peacock eye it would be at about $2 \mathrm{~m}(0.5 \mathrm{D})$. For the double peacock eye working with objects located at this distance or further, there is an effect that reminds the simultaneous double image, with one of them better focused than the other.

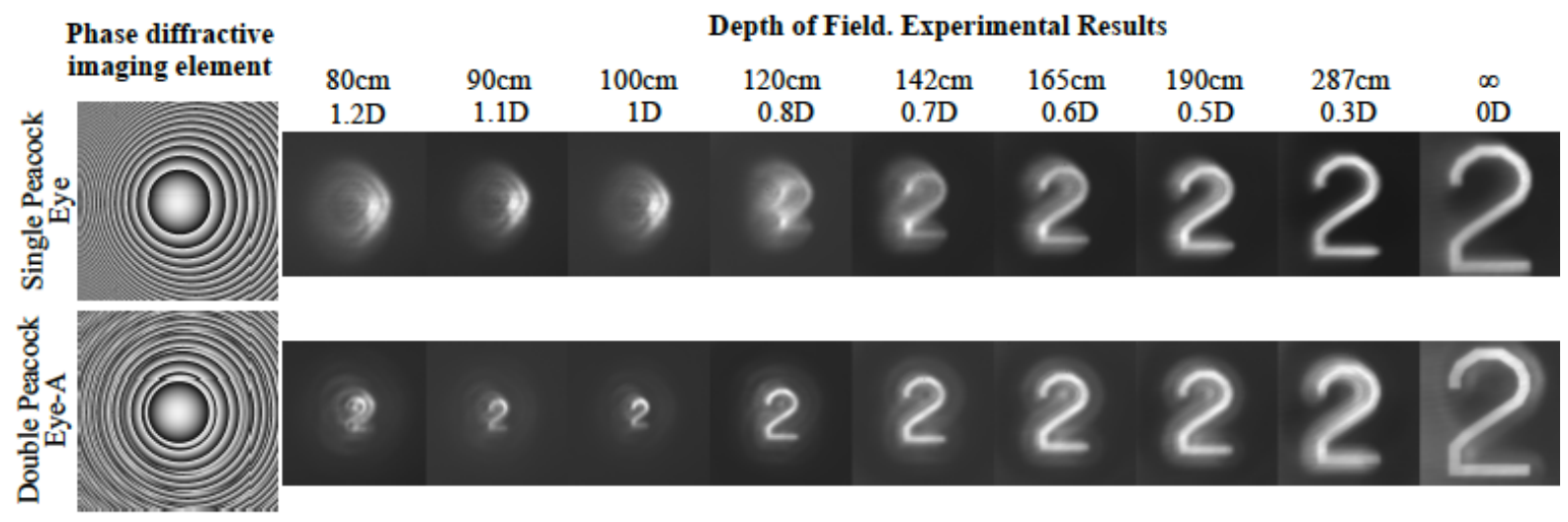

Figure 18. Experimental depth of field (object space) for two peacock eye base elements.

\section{Conclusions}

Ametropies can been compensated by means of programmed monofocal lenses displayed on the SLM. The use of programmable lenses allowed a fine-tuning procedure to obtain the necessary optical power for compensation in situ. The procedure consisted in sequentially reprogramming and refreshing the lenses on the modulator with a range of optical power values close to the initial calculated value. This was carried out to optimize the sharpness of the image on the retina of the artificial eye (the CCD sensor). As we have shown in the experimental results, this in situ fine tuning procedure of the programmable compensation lens has clear advantages over the conventional method of placing ophthalmic lenses of different optical power (with a ordinary precision not higher than of 0,25D) on a set of glasses or a phoropter. Certainly, with the use of an SLM it is possible to obtain a more precise, rapid, and comfortable compensation due to the fact that it does not require the changing of physical compensation lenses.

In the second stage, we have studied the generation of a PDOE that consists of a combination of three sublenses with different focal lengths and with optical axes in either coaxial or multi-axis configuration. In addition, for both configurations, we have used two methods for the spatial encoding of the phase distribution of the composite system: by rows, and randomly. We found the experimental results to be consistent with the numerical simulations carried out for each configuration. The experimental results bring out the fact that the multi-axis configuration (with separate parallel axis) is characterized by providing a good contrast in the images planes that are in focus. On the other hand, the coaxial 
configuration images with poor contrast due to the image overlapping on the same axis. Regarding the encoding methods, we have verified that the row encoding method generates multiorder image replicas given by the repeating pattern introduced by the codification, whereas the random encoding method has not this disadvantageous effect.

Finally, the experimental results presented in last stage prove that the PDOEs designed to focus an incident plane wave into a segment of the optical axis, satisfactorily perform as EDOF imaging components. We have implemented several PDOEs with EDOF such as: the Forward Logarithmic Axicon (FLAX), the Axilens (AXL), the Light Sword optical element (LSOE), the Peacock Eye (PE), and the double Peacock Eye (DPE) whose phase function is equivalent to the so-called progressive ophthalmic lens. Each PDOE has a focal segment that extends between the design focals $\left[f_{1}, f_{2}\right]$ and its performance has been compared to a programmable trifocal Fresnel lens. We have evaluated the quality of the image formed by each PDOE by means of the PSF and the MTF.

The image quality produced by all DOEs along the focal segment reveals that, with the exception of the trifocal lens, all elements are capable of extending smoothly the depth of focus. However, not all elements preserve the image quality in a significant way along the focal segment. The FLAX and AXL are the elements that perform worst with the image quality rapidly decreasing from the central part of the focal segment to the extreme of less optical power. Out of all the remaining DOEs, the LSOE and the DPEs are the ones that produce the best image quality along the focal segment. Nonetheless, these elements also display a loss of resolution and contrast. Therefore, the results suggest that a trade-off between the extended depth of focus and image sharpness should be achieved depending on the application. Moreover, these results open new avenues for the design of optical systems invariant to defocus oriented toward ophthalmic applications, like the compensation of presbyopia.

\section{Acknowledgements}

This study was partially supported by project DPI2013-43220-R from the Spanish Ministerio de Economía y Competitividad and FEDER. 